\title{
Temperature and composition evolution of lattice, charge density wave and superconducting orders in Ta-based dichalcogenides by total x-ray scattering
}

\author{
Valeri Petkov \\ Central Michigan University, Mount Pleasant, United States of America; \\ petkolvg@cmich.edu
}

\begin{abstract}
A characteristic feature of quantum materials is the presence of lattice degrees of freedom manifesting themselves as local structural distortions leading to competing ground state phases and exotic behavior. More often than not, the distortions are not well expressed and/or perfectly periodic, making it difficult to identify and quantify them using traditional crystallographic techniques. We will demonstrate the advantages of total x-ray scattering and large-scale structure modeling in studying the temperature and composition evolution of lattice instabilities related to the emergence of charge density wave (CDW) and superconducting (SC) orders in archetypal $\mathrm{Ta}(\mathrm{Se} / \mathrm{Te})_{2}$ quantum materials. In particular, we will show that the low-temperature CDW phase of hexagonal $2 \mathrm{H}-$ $\mathrm{TaSe}_{2}$ emerges via a gradual buildup of locally correlated clusters of Ta atoms, and not via a sudden onset of a Ta superstructure at the transition temperature [1]. We will also show the presence of a hierarchical relationship among the crystal lattice, CDW and SC orders in ternary Ta-Te-Se solid solutions, where different degrees of crystal lattice order appear to promote and maintain the competing CDW and SC orders to a different extent. The relationship may well explain the observed irregular evolution of the SC transition temperature with the relative Te to Se ratio in the solid solutions [2].
\end{abstract}

[1] V. Petkov et al. Phys. Rev. B 101, 121114(R) (2020).

[2] V. Petkov et al. Phys. Rev. B 103, 094101 (2021).

Keywords: total scattering, incomensurate crystals, transition metal dichalcogenides, charge density waves 